\title{
Influencia climática sobre la variación radial de caracteres anatómicos de madera en Abies religiosa
}

\author{
Sergio Bernal-Salazar ${ }^{1}$ \\ Teresa Terrazas Salgado ${ }^{1}$
}

\begin{abstract}
RESUMEN
Se caracterizó la variación radial de las dimensiones de las traqueidas y la identificación de las variables climáticas que influyen sobre la variación de las mismas en Abies religiosa (H.B.K.) Schldtl. \& Cham. Se eligieron 20 árboles en una población de la sierra El Campanario, Michoacán. Las muestras fueron tomadas a la altura del pecho $(1.30 \mathrm{~m})$, y procesadas con microtecnia convencional para madera. Se analizaron cuatro características de las traqueidas y el ancho de los anillos de crecimiento en sentido radial. Los diámetros tangenciales de la traqueida y del lumen de la traqueida en la madera tardía, así como del lumen de la traqueida en la madera temprana, presentan un ligero incremento que va de la médula al cámbium vascular. El grosor de la pared de la traqueida en la madera tardía muestra un rápido incremento en la región cercana a la médula y posterior a este incremento, manifiesta amplias fluctuaciones. El ancho de los anillos de crecimiento tiene un patrón inverso al grosor de la pared. Los análisis de regresión mostraron que las temperaturas máximas afectan la acumulación y las características de las traqueidas. Los resultados sugieren que la actividad del cámbium vascular de Abies religiosa está relacionada con las temperaturas máximas.
\end{abstract}

PALABRAS CLAVE:

Variación radial, traqueidas, Abies religiosa, México.

\begin{abstract}
The objective of this research was to characterize radial tracheid variation and to identify the climatic features that affect tracheid variation in Abies religiosa (H.B.K.) Schldtl. \& Cham.. Twenty trees of Abies religiosa were selected in a population at sierra El Campanario in the state of Michoacan. Wood samples were removed at $\mathrm{dbh}(1.30 \mathrm{~m})$ and prepared by the standard wood procedure. Four anatomical features of tracheids and ring width were analyzed. Tangential diameter of latewood tracheid and tangential lumen diameter of latewood tracheids, as well as tangential lumen diameter of earlywood tracheids show an increment from pith to vascular cambium. Latewood tracheid wall thickness has a strong increment near the pith, followed by amply fluctuations. Ring width possesses an inverse pattern than wall thickness. Regression analyses show that higher temperatures affect tracheid accumulation and size. These results suggest that maximum temperatures affect Abies religiosa cambial cells.
\end{abstract}

KEY WORDS:

Radial variation, tracheids, Abies religiosa, Mexico

1 Colegio de Posgraduados. Programa de Botánica. Km. 35.5 carr. México-Texcoco. Montecillo, Edo. de México, 56230 México.bernals@colpos.colpos.mx; winchi@colpos.colpos.mx.

Manuscrito recibido el 10 de noviembre de 1999. Aceptado el 28 de enero de 2000 


\section{INTRODUCCIÓN}

La formación y acumulación de la madera en los árboles da información que puede ser usada como registro de cambios ambientales. Las características de los anillos de crecimiento (ancho y densidad) y de los elementos traqueales (longitud, diámetro y grosor de la pared) revelan detalles de la historia natural de un individuo y del ecosistema (Jagels et al., 1994; Pumijumnong y Park, 1999), como por ejemplo el ataque de enfermedades $y$ plagas, el déficit hídrico prolongado y los daños causados por fuego (Shortle et al., 1995; Weber, 1997). Por ello, los estudios dendrocronológicos, basados en la variación radial de la madera de la médula al cámbium vascular y los parámetros ambientales, son importantes para entender el comportamiento de las especies leñosas que conforman una comunidad forestal (Vaganov, 1996; Wilpert, 1991; Wimmer et al., 1996).

Las fases de crecimiento y relativa inactividad en muchas plantas están estrechamente relacionadas con los cambios ambientales. Las bajas temperaturas usualmente inducen periodos de inactividad en los meristemos, mientras las condiciones húmedas y cálidas provocan su reactivación. Esta periodicidad en el cámbium vascular determina los patrones de producción y acumulación de los elementos traqueales que componen la madera (Ajmal e lqbal, 1987; Lim y Soh, 1997). En las especies que se desarrollan en ambientes tropicales e intertropicales el patrón de actividad cambial difiere considerablemente al de las especies de regiones templadas. En latitudes tropicales los árboles usualmente no presentan anillos de crecimiento distintivos y el cámbium vascular permanece activo todo el año o gran parte de éste (Dave y Rao, 1982; Borchert, 1999).
La variación de los elementos traqueales de la madera en relación con su posición radial respecto al eje del árbol está ampliamente reconocida para especies de zonas templadas. Panshin y De Zeeuw (1980) sintetizan las curvas de variación radial para algunas especies de bosques templados, sin embargo para la gran mayoría de las especies en México se desconocen los patrones de variación.

\section{OBJETIVOS}

Los objetivos de esta investigación fueron generar las curvas de variación radial para los caracteres anatómicos de las traqueidas y el ancho de los anillos de crecimiento e identificar las variables climáticas que influyen sobre la variación de los mismos en árboles de Abies religiosa.

\section{MATERIALES Y MÉTODOS}

La selección de árboles y recolección de muestras de madera se realizó en La Reserva Especial de la Biosfera Mariposa Monarca ubicada en la sierra EI Campanario en el Estado de Michoacán. La región se localiza entre los 19 $30^{\prime}$ y los $19^{\circ} 36^{\prime}$ de latitud norte y entre los $100^{\circ} 10^{\prime}$ y los $100^{\circ}$ 15 ' de longitud oeste (Fig. 1). La zona presenta un clima semifrío y subhúmedo con lluvias en verano. La precipitación media anual es de $1,228 \mathrm{~mm}$ y la temperatura media anual de $10.7^{\circ} \mathrm{C}$. La fórmula climática reportada para la zona corresponde al C (e) (w2) (w) (García, 1981). Fisiográficamente la zona es homogénea, con suelos profundos bien drenados, ácidos, derivados de cenizas volcánicas y clasificados como Andosol órtico. Los principales árboles que componen este bosque de oyamel son Abies religiosa, Pinus pseudostrobus y varias especies de Cupressus (Rzedowski, 1978). 


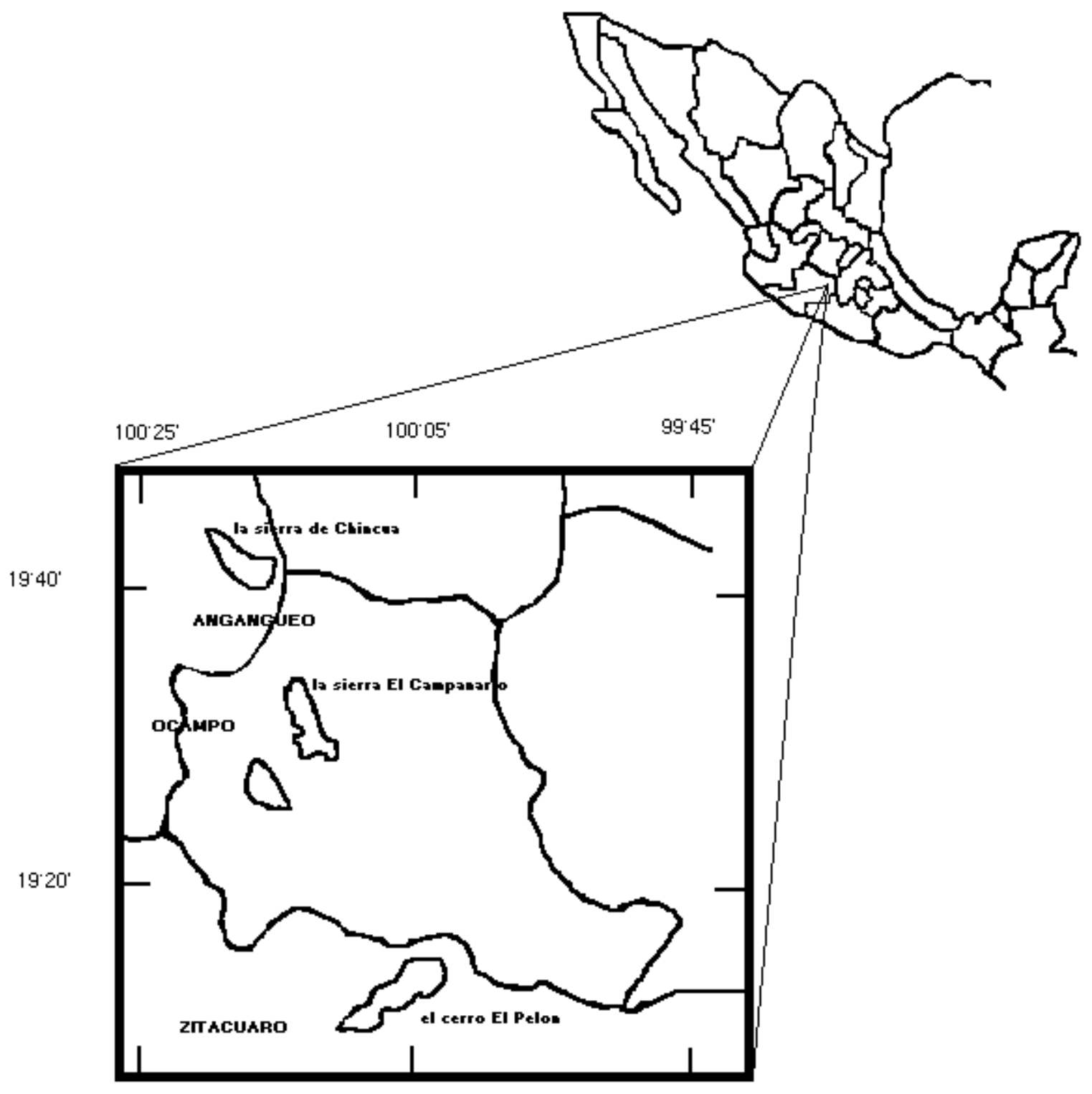

Figura 1. Localización del área de muestreo, la sierra El Campanario en el estado de Michoacán (modificado de Huante et al.,1991). 
Para la recolecta se establecieron cuatro sitios de muestreo ubicados entre los 3,300 y 3,500 msnm y con pendientes no mayores a 30/. Dentro de cada sitio se seleccionaron cinco árboles con un diámetro que varía de 19.57 a $33.10 \mathrm{~cm}$ a la altura del pecho (Tabla 1).

Se tomaron dos muestras por árbol a $1.30 \mathrm{~m}$ del suelo con un taladro de $12 \mathrm{~mm}$ de diámetro evitando la pendiente. Las muestras fueron etiquetadas y fijadas en formaldehído, ácido acético y alcohol etílico (Berlyn y Miksche, 1976) y después de $24 \mathrm{~h}$ se guardaron en glicerina, alcohol y agua (1:1:1) con objeto de evitar su secado. En el laboratorio las muestras se colocaron en soportes para cuantificar el número de los anillos de crecimiento. Se hicieron cortes transversales de $30 \mu \mathrm{m}$ de grosor con un micrótomo de deslizamiento; estos se deshidrataron en cambios de alcohol etílico, tiñeron con safranina y se montaron con resina sintética (Berlyn y Miksche, 1976).

En las preparaciones de los cortes transversales se realizaron 25 mediciones en cada anillo de crecimiento para el diámetro tangencial de la traqueida en la madera tardía, el diámetro tangencial del lumen de la traqueida en la madera tardía, el diámetro tangencial del lumen de la traqueida en la madera temprana y el grosor de la pared de la traqueida en la madera tardía. En cada anillo se seleccionaron las traqueidas a medir entre las segunda y la sexta célula antes y después de la zona de transición entre la madera tardía y temprana. También se midió el ancho de los anillos de crecimiento en milímetros. Las mediciones se realizaron con un analizador de imágenes Image-Pro® Plus v. 3.0 (Media Cybernetics, 1997) adaptado a una cámara de video Hitachi KP-D51 y a un microscopio Olympus BX-50.

Las variables independientes fueron los datos mensuales de temperatura máxima, mínima y promedio, así como precipitación total. Los datos climáticos se recopilaron del Servicio Meteorológico Nacional para la estación meteorológica Palizada y corresponden al periodo 19611997. Las variables dependientes fueron las cuatro características anatómicas de las traqueidas y el ancho de los anillos de crecimiento.

Los análisis estadísticos se realizaron con el paquete estadístico SAS (SAS Institute, 1988). Un análisis (PROC MEANS) permitió obtener los estadísticos básicos con los cuales se construyeron las curvas de variación radial, éste fue seguido de un análisis exploratorio descriptivo (PROC UNIVARIATE), el cual permitió conocer que las variables anatómicas se ajustaban a una distribución normal. Para identificar y cuantificar la relación entre las variables climáticas y anatómicas se aplicaron varios análisis de regresión múltiple paso a paso (PROC REG).

Tabla 1. Diámetro a la altura del pecho $(1.30 \mathrm{~m})$ los árboles muestreados de Abies religiosa en la sierra El Campanario.

\begin{tabular}{|c|c|c|c|c|c|c|c|}
\hline $\begin{array}{c}\text { No. } \\
\text { ÁRBOL }\end{array}$ & $\begin{array}{l}\text { DIÁMETRO } \\
(\mathrm{cm})\end{array}$ & $\begin{array}{l}\text { No. } \\
\text { ÁRBOL }\end{array}$ & $\begin{array}{c}\text { DIÁMETRO } \\
(\mathrm{cm})\end{array}$ & $\begin{array}{l}\text { No. } \\
\text { ÁRBOL }\end{array}$ & $\begin{array}{c}\text { DIÁMETRO } \\
(\mathrm{cm})\end{array}$ & $\begin{array}{l}\text { No. } \\
\text { ÁRBOL }\end{array}$ & $\begin{array}{c}\text { DIÁMETRO } \\
(\mathrm{cm})\end{array}$ \\
\hline \multicolumn{2}{|c|}{ Sitio Uno } & \multicolumn{2}{|c|}{ Sitio Dos } & \multicolumn{2}{|c|}{ Sitio Tres } & \multicolumn{2}{|c|}{ Sitio Cuatro } \\
\hline 1 & 23.71 & 6 & 19.57 & 11 & 24.19 & 16 & 30.71 \\
\hline 2 & 27.37 & 7 & 20.53 & 12 & & 17 & 33.10 \\
\hline 3 & 22.91 & 8 & 21.32 & 13 & & 18 & 29.6 \\
\hline 4 & 25.46 & 9 & 22.28 & 14 & 19.89 & 19 & 20.21 \\
\hline 5 & 23.23 & 10 & 19.89 & 15 & 28.64 & 20 & 19.09 \\
\hline
\end{tabular}




\section{RESULTADOS}

Variación anatómica radial. En las figuras 2 y 3 se presentan los patrones de variación radial para los cuatro caracteres anatómicos de las traqueidas estudiados y el ancho de los anillos de crecimiento. Los árboles estudiados tuvieron un número máximo de 50 anillos.

El diámetro tangencial de la traqueida en la madera tardía y el diámetro tangencial del lumen de la traqueida en la madera tardía, así como el diámetro tangencial del lumen de la traqueida en la madera temprana presentan un ligero incremento de la médula al cámbium vascular (Fig. 2). Esto es, el incremento en el diámetro tangencial de la traqueida en la madera tardía (DITRA) es de $34.04: \mathrm{m}$ cerca de la médula a 50.40 $: \mathrm{m}$ próximo a las células del cámbium vascular. Mientras el diámetro tangencial del lumen de la traqueida en la madera tardía (DILUTRA) se incrementa de 23.10 a 32.79 : $\mathrm{m}$ en el mismo sentido y para el diámetro tangencial del lumen de la traqueida en la madera temprana (DILUMT) aumenta de 27.86 a $42.54: \mathrm{m}$. A partir de los 27 años se presentan ligeras fluctuaciones en las dimensiones del diámetro (Fig. 2).

El grosor de la pared de la traqueida en la madera tardía (GRTRA) muestra un rápido incremento en los primeros 25 anillos de crecimiento de 5.46 a $7.89: \mathrm{m}$. Sin embargo, a partir del anillo 25 existen mayores fluctuaciones hasta alcanzar un grosor de $9.4: \mathrm{m}$ (Fig. 3).

El ancho de los anillos de crecimiento (ANCRE) muestra una tendencia inversa a la del grosor de la pared de la traqueida en la madera tardía, reduciéndose de un valor de $5.65 \mathrm{~mm}$ cerca de la médula a $1.12 \mathrm{~mm}$ próximo al cámbium vascular (Fig. 3).

Relación clima-caracteres anatómicos y ancho del anillo de crecimiento. Los resultados del análisis de regresión múltiple indican que en la sierra El Campanario, para el periodo de 1961-1997, las variables meteorológicas que contribuyen a la predicción de las variables anatómicas son la temperatura máxima de marzo, julio y octubre (Tabla 2), mientras que las temperaturas mínimas y promedio, así como la precipitación total no contribuyen a la predicción de las variables anatómicas o ésta es muy baja (Tabla 2). Los coeficientes de determinación $\left(R^{2}\right)$ indican que el $99.65 \%$ de la variabilidad del diámetro tangencial de la traqueida de la madera tardía (DITRA), el $99.48 \%$ para el diámetro tangencial del lumen de la madera tardía (DILUTRA) y el $99.61 \%$ para el diámetro tangencial del lumen de la traqueida en la madera temprana (DILUMT) son explicadas por la temperatura máxima de octubre (22.40/C, promedio de la temperatura máxima, TPM), respectivamente (Tabla 2). En la figura 4 se muestra la relación entre las variables anatómicas DITRA, DILUTRA y DILUMT con la temperatura máxima de octubre para los años de 1961 a 1997.

Para el grosor de la pared de la traqueida de la madera tardía (GRTRA) el $98.93 \%$ de la variabilidad se explica por la temperatura máxima de julio (21.70/C TPM), mientras que la contribución de las variables temperatura máxima de abril y precipitaciones totales de enero y febrero son muy bajas (Tabla 2). En la figura 5 se presenta el grosor de la pared de la traqueida en la madera tardía contra la temperatura máxima de julio. Existe una alta correspondencia entre los picos máximos de temperatura y los del grosor de la pared. Para el ancho de los anillos de crecimiento (ANCRE) el $77.06 \%$ de la variabilidad se explica por la temperatura máxima de marzo (25.91/C TPM) (Tabla 2) y una baja contribución se atribuye a la precipitación total de enero, por lo que hay cerca de un $22 \%$ de la variación que no es explicada por las variables ambientales incluidas en el análisis. La relación entre la temperatura máxima de marzo y el ancho de los anillos de crecimiento difiere en algunos años (Fig. $6)$. 


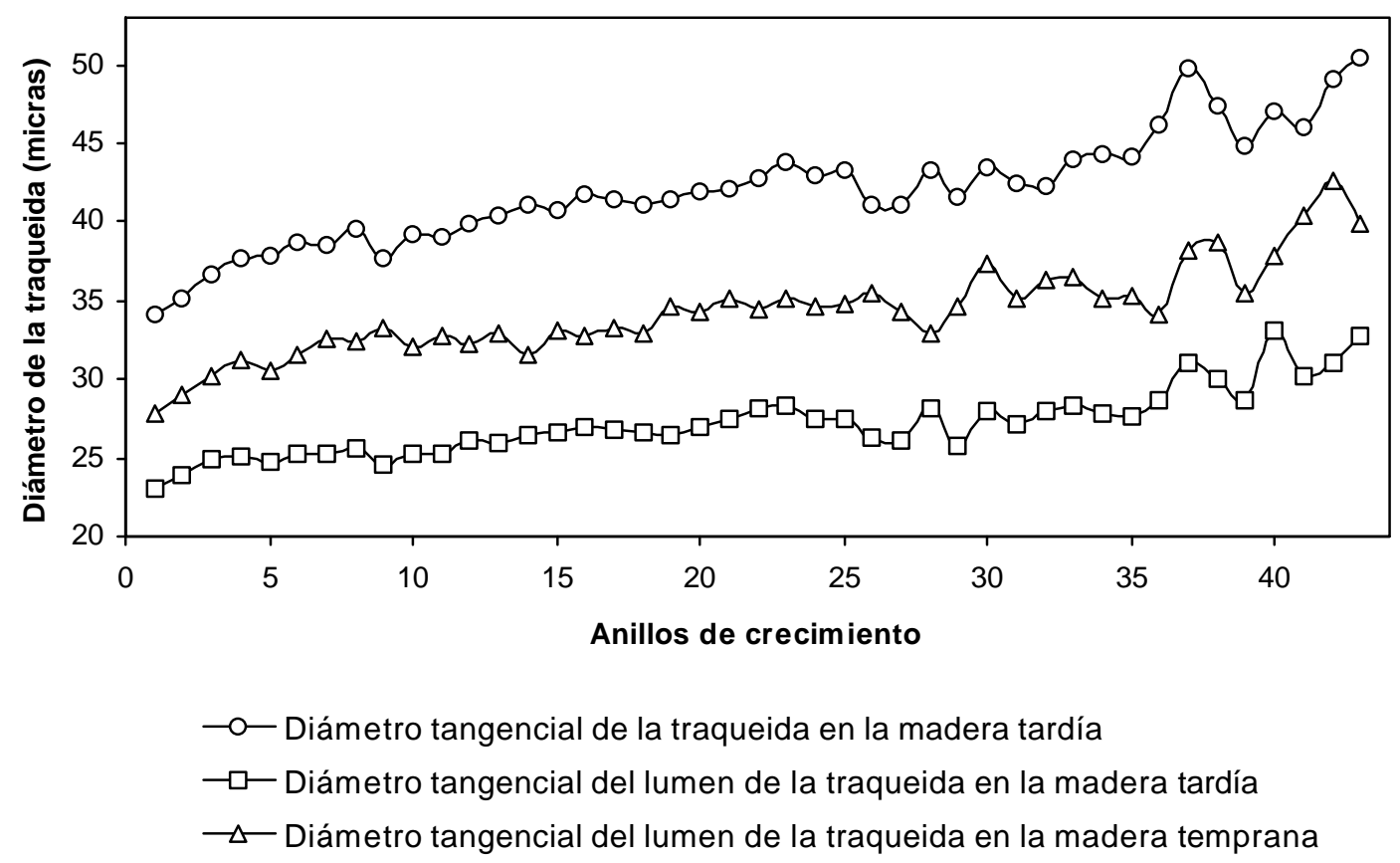

Figura 2. Variación radial del diámetro tangencial de la traqueida en la madera tardía, del diámetro tangencial del lumen de la traqueida en la madera tardía, y del diámetro tangencial del lumen de la traqueida en la madera temprana de la médula ( 0 anillos) hacia el cámbium vascular ( $>40$ anillos).

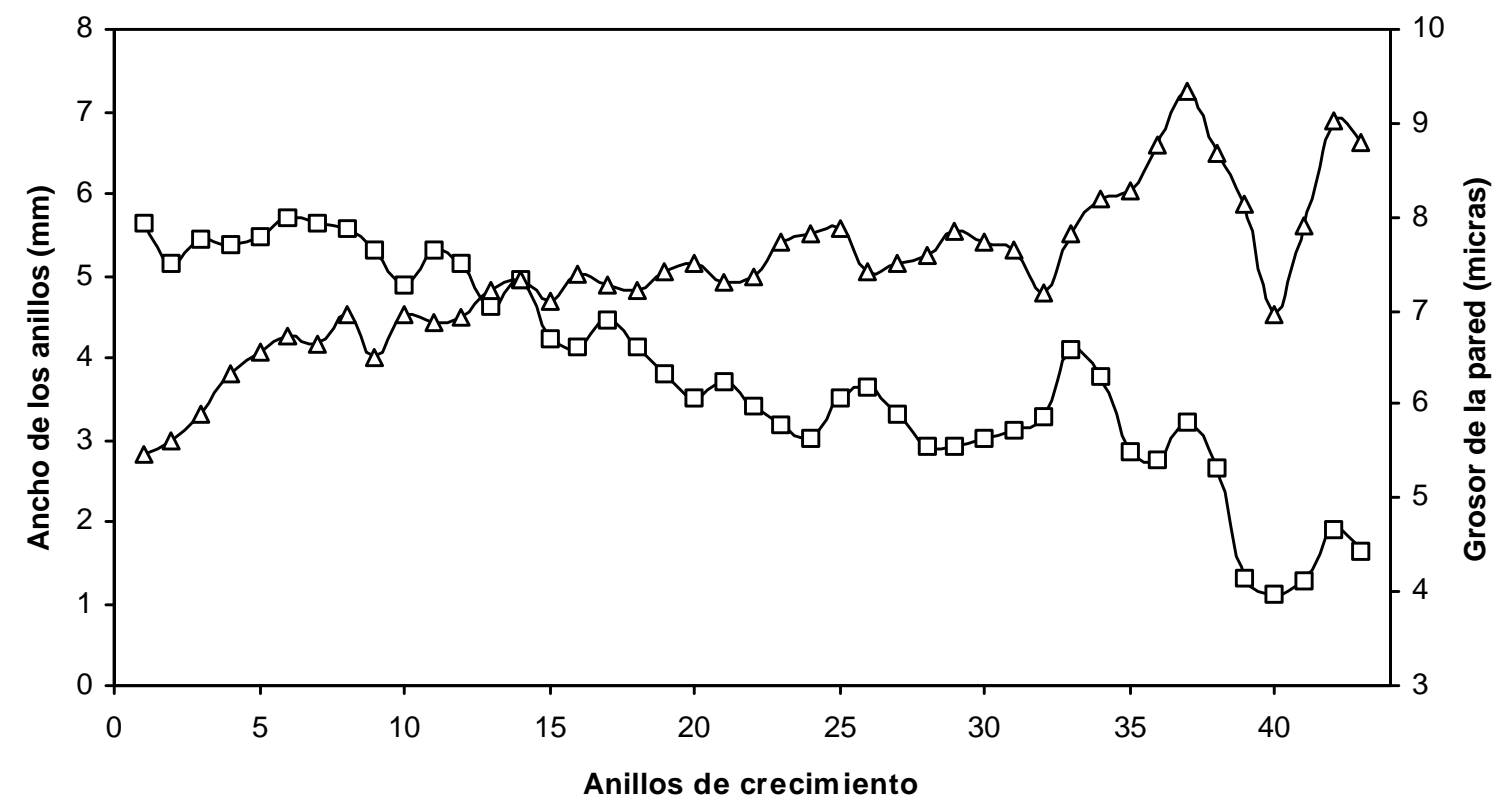

$\neg$ Ancho de los anillos de crecimiento $\neg-$ grosor de la pared de la traqueida en la madera tardía

Figura 3. Variación radial del grosor de pared de la traqueida en la madera tardía y del ancho de los anillos de crecimiento de la médula ( 0 anillos) hacia el cámbium vascular (> 40 anillos). 
Cuadro 2. Regresión múltiple (stepwise) entre las variables anatómicas y las variables meteorológicas para la sierra El Campanario (1961-1997). Únicamente se muestras aquellas variables meteorológicas con $\mathrm{R}^{2}>$ de 0.0001

\begin{tabular}{|c|c|c|c|c|}
\hline \multirow{2}{*}{\multicolumn{2}{|c|}{ VARIABLES }} & \multicolumn{3}{|c|}{ ESTIMADORES DE REGRESIÓN } \\
\hline & & $b$ & $\mathrm{R}^{2}$ & C.V \\
\hline DITRA & $\begin{array}{l}\text { T/ máx. Octubre } \\
\text { T/ máx. Junio } \\
\text { T/ máx. Marzo }\end{array}$ & 12.81 * & $\begin{array}{l}0.9984 \\
0.9965 \\
0.0010 \\
0.0006\end{array}$ & 3.2149 \\
\hline DILUTRA & $\begin{array}{l}\text { T/ máx. Octubre } \\
\text { T/ máx. Junio } \\
\text { T/ máx. Marzo }\end{array}$ & 11.35 * & $\begin{array}{l}0.9965 \\
0.9948 \\
0.0010 \\
0.0006\end{array}$ & 3.7554 \\
\hline DILUMT & $\begin{array}{l}\text { T/ máx. Octubre } \\
\text { T/ máx. Abril } \\
\text { T/ máx. Marzo } \\
\text { T/ máx. Junio }\end{array}$ & 10.24 * & $\begin{array}{l}0.9978 \\
0.9961 \\
0.0010 \\
0.0004 \\
0.0003\end{array}$ & 5.2291 \\
\hline GRTRA & $\begin{array}{l}\text { T/ máx. Julio } \\
\text { T/ máx. Abril } \\
\text { Prec. Enero } \\
\text { T/ med. Julio } \\
\text { Prec. Febrero }\end{array}$ & 4.09 * & $\begin{array}{l}0.9911 \\
0.9893 \\
0.0009 \\
0.0004 \\
0.0004 \\
0.0002\end{array}$ & 9.1253 \\
\hline ANCRE & $\begin{array}{l}\text { T/ máx. Marzo } \\
\text { Prec. Enero }\end{array}$ & 15.46 ** & $\begin{array}{l}0.7754 \\
0.7706 \\
0.0048\end{array}$ & 53.4746 \\
\hline
\end{tabular}

${ }^{\star}=p<0.0001,{ }^{* *}=p<0.005$

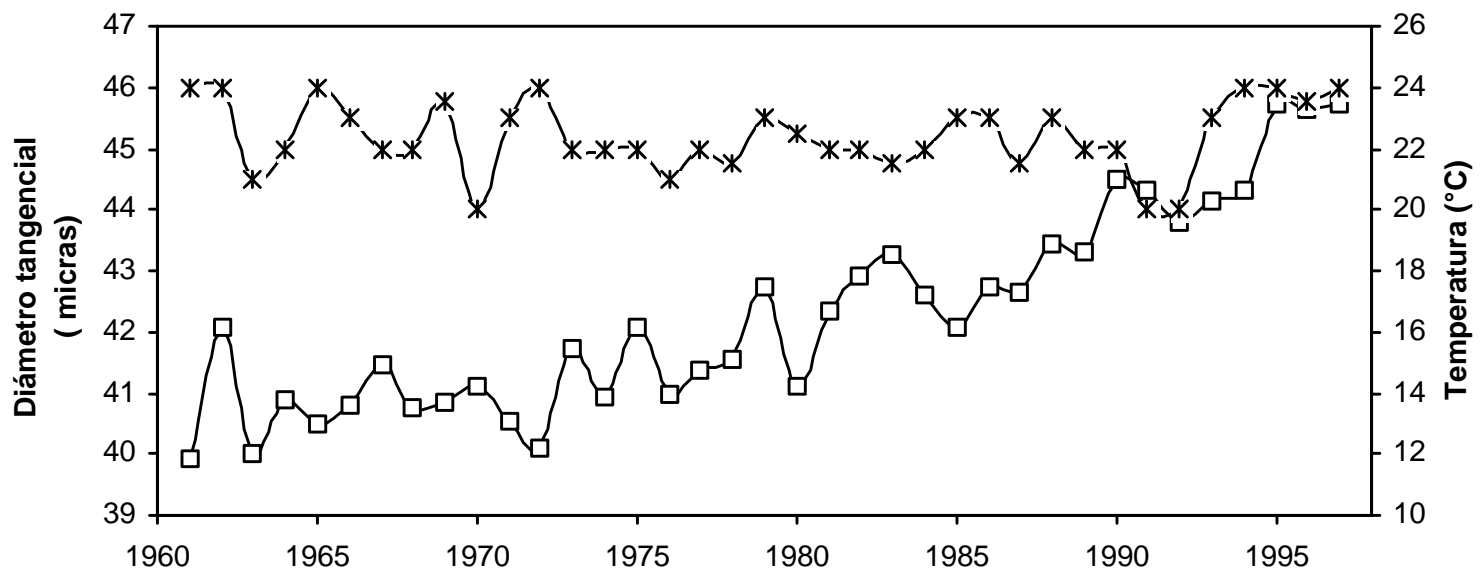

$\rightarrow \square$ Diámetro tangencial de la traqueida en la madera tardía $\rightarrow *-$ Temperatura máxima de octubre

Figura 4A. Relación temperatura máxima de octubre y las variables anatómicas de acuerdo con los análisis de regresión. Diámetro tangencial de la traqueida en la madera temprana. 


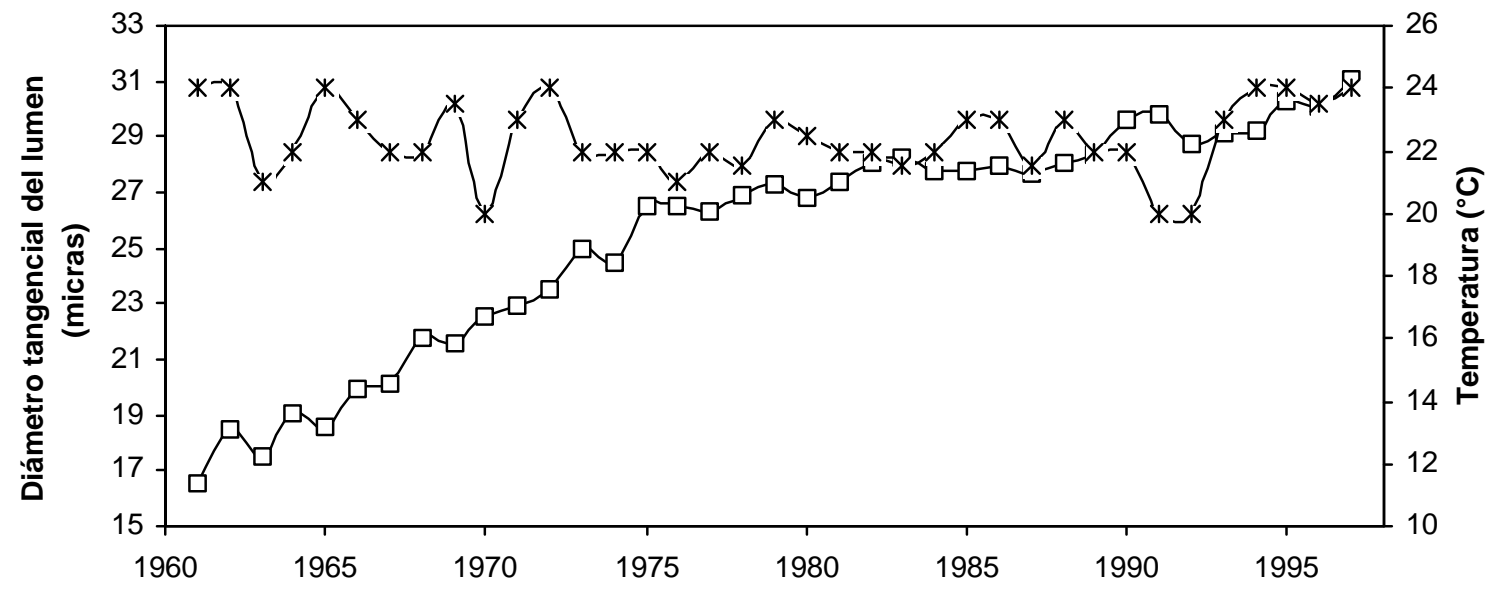

$\neg \square$ Diámetro tangencial del lumen de la traqueida en la madera tardía

-*-Temperatura máxima de octubre

Figura 4B. Relación temperatura máxima de octubre y las variables anatómicas de acuerdo con los análisis de regresión. Diámetro tangencial del lumen de la traqueida en la madera tardía.

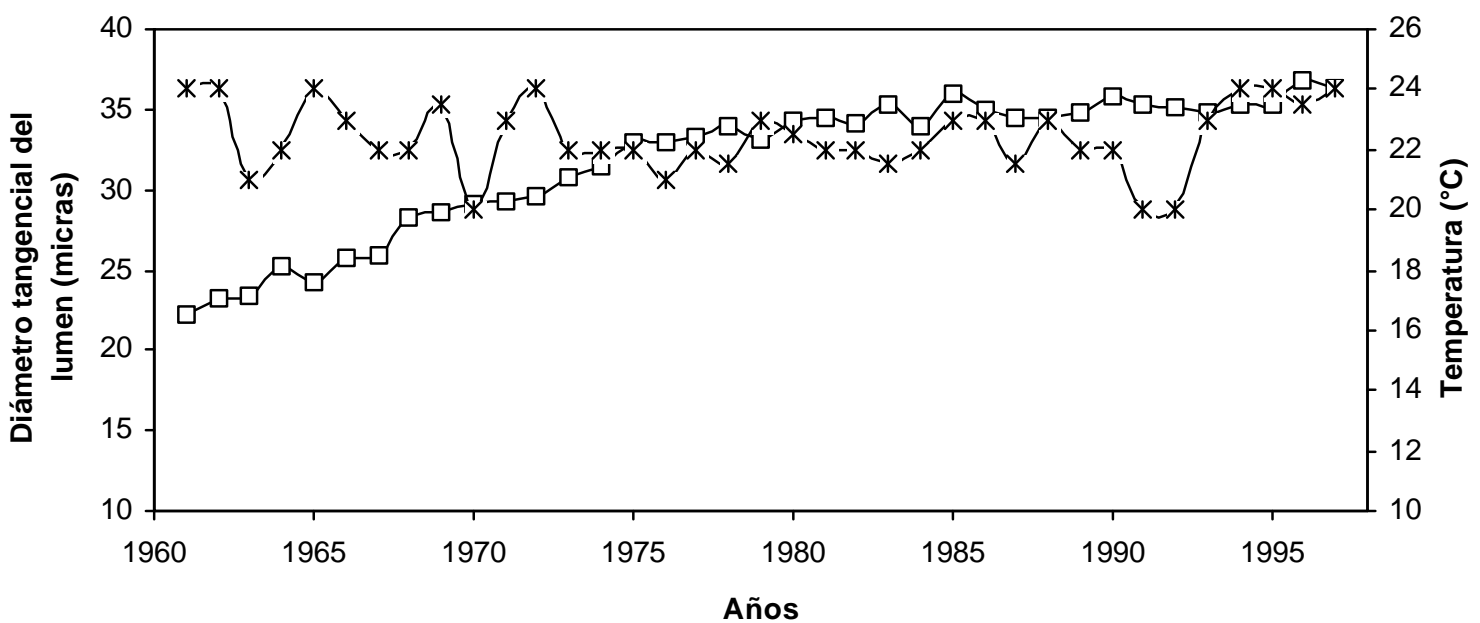

$\rightarrow$ Diametro tangencial del lumen de la traqueida en la madera temprana -*-Temperatura máxima de octubre

Figura 4C. Relación temperatura máxima de octubre y las variables anatómicas de acuerdo con los análisis de regresión. Diámetro tangencial del lumen de la traqueida en la madera temprana. 


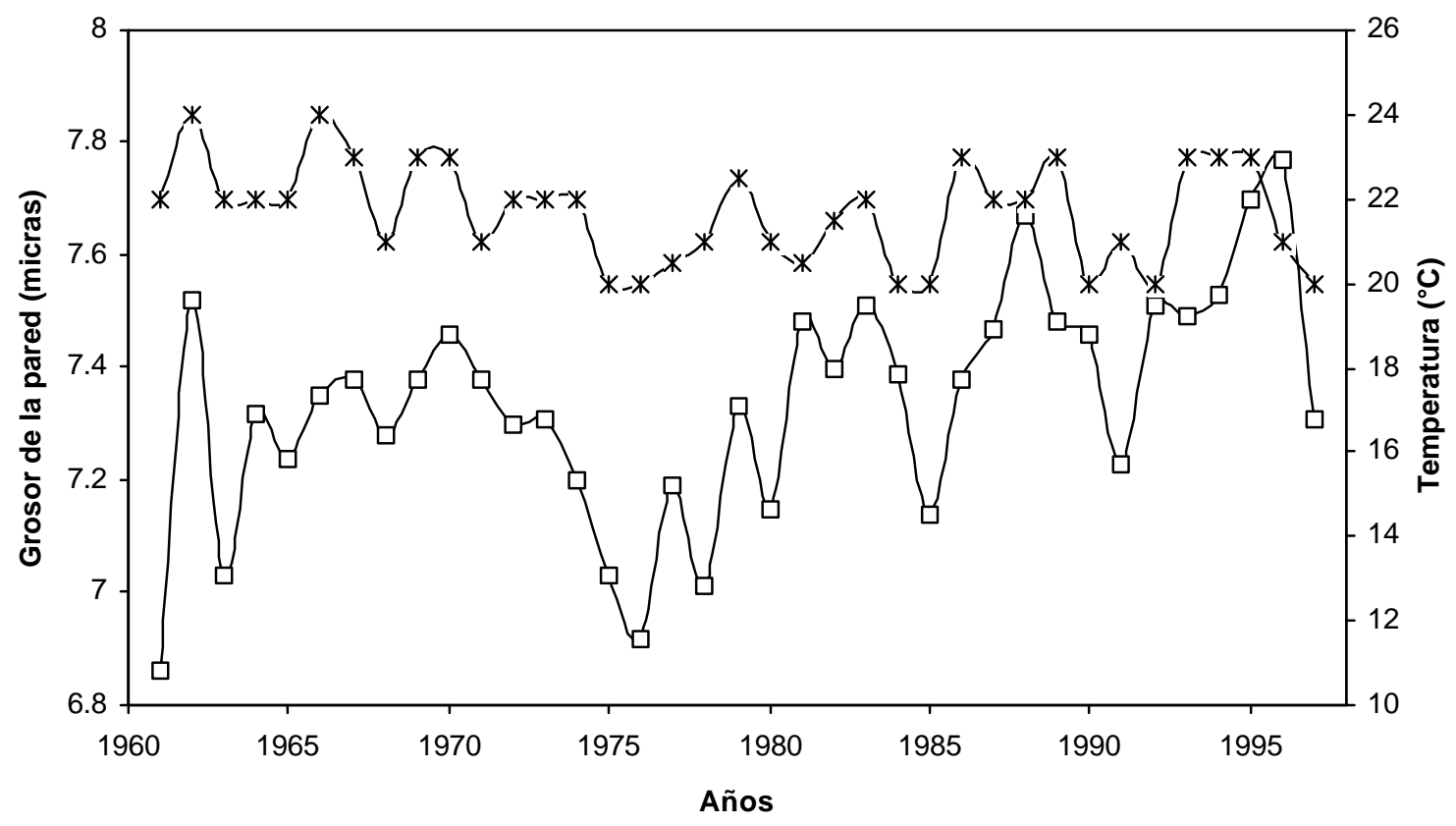

$\neg-$ Grosor de la pared de la traqueida en la madera tardía $-*-$ Temperatura máxima de julio

Figura 5. Relación de la temperatura máxima de julio con el grosor de la pared de la traqueida en la madera tardía de acuerdo con el análisis de regresión.

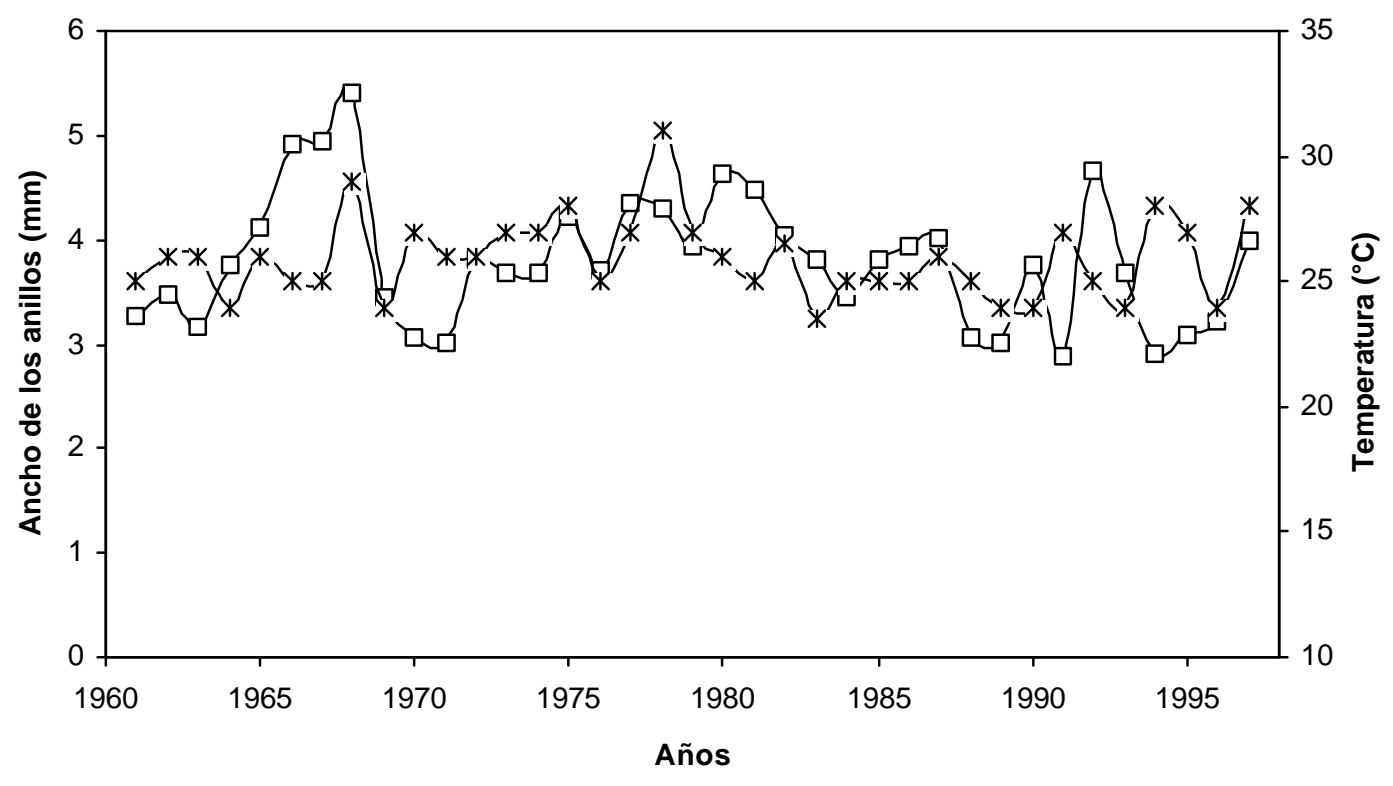

$\multimap$ - Ancho de los anillos de crecimiento $-*-$ Temperatura máxima de marzo

Figura 6. Relación de la temperatura máxima de marzo con el ancho de los anillos de crecimiento de acuerdo con el análisis de regresión. 


\section{DISCUSIÓN}

Variación anatómica radial. Las curvas de variación radial para los caracteres anatómicos de la madera pueden ser divididas en dos fases. La fase inicial, localizada cerca de la médula muestra un rápido incremento en la talla celular, especialmente en la longitud y el grosor de la pared, asociado con el periodo de maduración cambial. Mientras que la segunda fase, corresponde al periodo de estabilidad del cámbium vascular, donde las dimensiones celulares alcanzan su mayor talla y los cambios se dan por crecimiento intrusivo (Panshin y De Zeeuw, 1980). El comportamiento de las dimensiones de las traqueidas para los árboles de Abies religiosa muestra que los individuos estudiados no han alcanzado la maduración cambial 0 bien siguen un patrón de crecimiento indeterminado como Abies pindrow, Pinus ponderosa y Pinus strobus (Panshin y De Zeeuw, 1980). Con objeto de corroborar cuál de los dos patrones de maduración cambial presenta Abies religiosa se sugiere tomar muestras de árboles con un mayor diámetro y consecuentemente mayor edad.

La variación radial para el diámetro tangencial de la traqueida en la madera tardía, el diámetro tangencial del lumen de la traqueida en la madera tardía y el diámetro tangencial de la traqueida de la madera temprana presentan patrones similares de incremento de la médula hacia el cámbium vascular. Un comportamiento similar de variación fue reportado para Pinus radiata (Cown, 1975), Pinus pinea (Kasir y Al-Hialy, 1989), Cupressus sempervirens (Paraskevopoulou, 1991) y Picea sitchensis (Mitchell y Denne, 1997). El incremento en el diámetro de las traqueidas de la médula hacia el cámbium vascular puede interpretarse como una respuesta a la demanda conductiva de la planta debido a una mayor talla. Un mayor diámetro en la traqueida en la madera temprana es una respuesta a la función principal que desempeña: el transporte; la madera tardía, por su parte, cumple dos funciones: el transporte y la resistencia mecánica, por lo que un diámetro reducido junto con una pared gruesa puede evitar embolismos en las traqueidas.

El grosor de la pared de la traqueida en la madera tardía de Abies religiosa presenta un incremento rápido a partir de la médula y fluctúa hacia el cámbium vascular. Un patrón similar se encontró en Criptomeria japonica (Ohta, 1972). La fuerza mecánica que ofrecen las traqueidas se relaciona principalmente con el grosor de la pared. El aumento en esta característica de la médula hacia el cámbium vascular proporciona un soporte adecuado para el crecimiento del árbol.

El ancho de los anillos de crecimiento mostró una tendencia inversa al grosor de la pared de la traqueida. La reducción en el ancho de los anillos está fuertemente relacionada con la actividad del cámbium vascular. En la región juvenil, cerca de la médula, la proporción de las divisiones anticlinales es mayor que las periclinales, favoreciendo el incremento del diámetro del tronco rápidamente. Sin embargo, en la región de la madera madura la proporción de divisiones anticlinales/periclinales se invierte favoreciendo el incremento en circunferencia (Siddiqi, 1991). En especies de gimnospermas que crecen en latitudes mayores a las de México la variación en el ancho de los anillos tiende a ser baja, sin embargo este patrón puede ser modificado por factores ambientales o metabólicos (Larson, 1994), como posiblemente sea el caso de los árboles de Abies religiosa para esta región.

Relación clima-caracteres anatómicos y ancho del anillo de crecimiento. El periodo de actividad del cámbium vascular está determinado por los factores climáticos en donde se desarrollan las especies. En la región intertropical la actividad del cámbium vascular se presenta en la mayor parte del año debido a las condiciones de humedad y temperatura; pero en las zonas desérticas, 
la actividad se restringe al periodo de lluvias y en las zonas templadas se inicia cuando las temperaturas aumentan (Borchert, 1999; Creber y Chaloner, 1984). En la sierra El Campanario las temperaturas máximas de marzo, julio y octubre son las predictoras de las cuatro variables anatómicas y del ancho de los anillos de crecimiento para los árboles de Abies religiosa. Estos resultados concuerdan con lo reportado para Tectona grandis y Halocarpus biformis donde las características de la madera se correlacionan con las temperaturas (Pumijumnong y Park, 1999; Xiong et al., 1998). Huante et al. (1991) en un estudio dendrocronológico con Abies religiosa en Michoacán también encuentran una fuerte asociación entre el ancho de los anillos de crecimiento y la temperatura máxima de octubre y noviembre. Los resultados de este trabajo y los de Huante et al. (1991) sugieren que la actividad del cámbium vascular de Abies religiosa es de marzo a octubre o hasta noviembre y posiblemente su reactivación está determinada por el cambio en la temperatura, tal como sucede en las especies de zonas templadas (Creber y Chaloner, 1984).

Los reportes para varias especies que habitan en las regiones tropicales e intertropicales indican que la actividad de su cámbium vascular tiene una mayor amplitud a lo largo del año con respecto a las especies de zonas templadas (Ajmal e lqbal, 1987; Siddiqi, 1991; Liphschitz et al., 1984). Liphschitz et al. (1984) reportan la actividad cambial para dos especies de pinos que crecen en clima mediterráneo. Para Pinus halapensisseñalan dos periodos de actividad en el cámbium vascular, en primavera y otoño y de inactividad en verano e invierno; mientras que Pinus pinea mantiene una actividad cambial de abril a noviembre. Los autores mencionan que Pinus halapensis se adapta al clima mediterráneo, entrando en inactividad cambial en el periodo de sequía, mientras que Pinus pinea sigue el ritmo de actividad del cámbium vascular de las regiones templadas, ampliando la duración de su actividad. En este sentido el cámbium vascular de los individuos de Abies religiosa en la región estudiada, posiblemente permanece activo durante gran parte del año, como una consecuencia de las condiciones climáticas favorables en la zona.

Las fase de actividad e inactividad del cámbium vascular han sido divididas en tres periodos; el primero es cuando las células están activas metabólicamente pero no se dividen. En este periodo existe almacenamiento y movilización de fotosintatos en las células del parénquima. El segundo periodo es cuando las células se dividen activamente y por último cuando las células no muestran actividad metabólica ni divisiones (lqbal, 1995). Ajmal e lqbal (1987) reportan que la actividad del cámbium vascular en Streblus asper se inicia a principios de mayo y termina a mediados de noviembre. Sin embargo, la división celular que da origen al xilema comienza en julio y finaliza a mediados de noviembre. Para Ficus religiosa, el cámbium vascular produce células de xilema y floema de agosto a finales de diciembre y la formación de células precursoras se presenta en marzo (Siddiqi, 1991). Resultados similares se han reportado para Dalbergia sissoo, Polyalthia longifolia y Callistemon citrinus (lqbal, 1991). Los resultados de esta investigación sugieren que la actividad del cámbium vascular de Abies religiosa puede ser similar a lo reportado para otras especies con tres periodos de actividad y producción de xilema en la fase de división activa.

La actividad del cámbium vascular se asocia estrechamente con la cantidad de fotosintatos disponibles y el metabolismo hormonal del árbol; y estos con el fotoperiodo, la disponibilidad de agua y la temperatura que modifican, en conjunto, la tasa fotosintética en los árboles (Larson, 1963; Torelli et al., 1986; Venogupal y Krishnamurthy, 1987). El grosor de la pared de la traqueida depende de la cantidad de los carbohidratos disponibles y 
almacenados en las células del parénquima y posiblemente la acumulación de la pared en las traqueidas para los árboles de Abies religiosa se inicie en agosto y por lo tanto el grosor estará determinado por la cantidad de carbohidratos disponibles.

El ancho de los anillos de crecimiento está influenciado por la temperatura máxima de marzo. Este mes podría ser el momento de la producción de células precursoras del xilema y floema, la cantidad de dichas células determinará la producción de la madera y por consiguiente el ancho de los anillos de crecimiento. Sin embargo, las variables ambientales incluidas en el análisis explican menos del $80 \%$ de la variación, por lo que otras variables biológicas (vigor, competencia) o climáticas (humedad relativa, fotoperiodo) también influyen en la acumulación de madera.

\section{CONCLUSIONES}

Los caracteres anatómicos analizados para Abies religiosa presentan patrones de maduración similares a los reportados para otras gimnospermas. La variación radial para el diámetro tangencial de la traqueida en la madera tardía, el diámetro tangencial del lumen de la traqueida en la madera tardía y el diámetro tangencial del lumen de la traqueida en la madera temprana presenta un incremento progresivo de la médula al cámbium vascular. El grosor de la pared de la traqueida en la madera tardía muestra un rápido incremento en la región cercana a la médula y posterior a este incremento manifiesta amplias fluctuaciones. El ancho de los anillos de crecimiento tiene un patrón inverso al grosor de la pared de la traqueida.

Los análisis de regresión mostraron que las temperaturas máximas afectan el ancho de los anillos de crecimiento y las características de las traqueidas en los individuos de Abies religiosa en la zona estudiada. Se sugiere que el periodo de actividad del cámbium vascular de Abies religiosa está influenciado por las temperaturas máximas del sitio y que el tiempo de actividad cambial es similar al reportado para otras especies que habitan las zonas intertropicales. Esta interpretación debe ser validada con estudios de actividad del cámbium vascular para Abies religiosa con el objeto de corroborar la influencia de la temperatura máxima en ella.

\section{RECONOCIMIENTOS}

Este artículo forma parte de la tesis de maestría realizada por el primer autor en el Colegio de Posgraduados, México, con apoyo otorgado por el CONACYT a través de la beca 114367 / 1997-1998. Se agradecen los comentarios y sugerencias de dos revisores anónimos para mejorar el manuscrito.

\section{REFERENCIAS}

Ajmal, S. y M. lqbal. 1987. Annual rhythm of cambial activity in Streblus asper. IAWA Bulletin new series 8(3): 275283.

Berlyn, G.P. y J.P. Miksche. 1976. Botanical microtechnique and cytochemistry. The lowa State University Press, Ames. 326 p.

Borchert, R. 1999. Climatic periodicity, phenology y cambium activity in tropical dry forest trees. IAWA Journal 20(3): 239-247.

Cown, D.J. 1975. Variation in tracheid dimensions in the stem of a 26 year old Radiata Pine tree. Appita 28(4): 237-245.

Creber, G.T. y W.G. Chaloner. 1984. Influence of environmental factors on the wood structure of living and fossil trees. The Botanical Review 50(4) 357448. 
Dave, Y.S. y K.S. Rao. 1982. Seasonal activity of the vascular cambium in Gmelina arborea Roxb. IAWA Bulletin new series 3(1): 59-65.

García, E. 1981. Modificaciones al sistema de clasificación climática de Köppen (para adaptarlo a las condiciones de la República Mexicana). Instituto de Geografía, UNAM. México. 246 p.

Huante, P., E. Rincón y T.W. Swetnam. 1991. Dendrochronology of Abies religiosa in Michoacán, México. Treering Bulletin 51: 15-27.

lqbal, M. 1991. Structural and behavioral specialities of the vascular cambium of seed plants. In: Growth pattern of vascular plants. M. lqbal (Ed). Dioscorides Press, Portland, EUA. p: 211-271.

lqbal, M. 1995. Structure and behaviour of vascular cambium and mechanism and control of cambial growth. In: The cambial derivates. Ed. M. Iqbal. Bontraeger, Stuttgart, Berlin. pp: 1-67.

Jagels, R., J. Hornbeck y S. Marden. 1994. Drought and cold stress-induced morphometric changes in tree rings of red spruce. Technical Bulletin Maine Agricultural and Forest Experiment Station. 19 p.

Kasir, W.A. y A.S.A. Al-Hialy, 1989. Comparative study of some anatomical properties of four pines for using in pulp making. Mesopotamia Journal of Agriculture 21: 191-207.

Larson, P.R. 1963. The indirect effect of drought on tracheid diameter in red pine. Forest Science 9: 52-62.

Larson, P.R. 1994. The vascular cambium, development and structure. SpringerVerlag. Berlin. $725 \mathrm{p}$.
Lim, D.H. y W.Y. Soh. 1997. Cambial development and tracheid length of dwarf pines (Pinus densiflora and $P$. thunbergii). IAWA Journal 18(3): 301310.

Liphschitz, N., S. Lev-Yadun, E. Rosen y Y. Waisel. 1984. The annual rhythm of activity of the lateral meristem (cambium and phellogen) in Pinus halapensis Mill, and Pinus pinea $\mathrm{L}$. IAWA Bulletin new series 5(4): 263274.

Media Cybernetics. 1997. Image-Pro® Plus reference guide version 3.01 for Windows $^{\mathrm{TM}}$. Maryland. EUA. 480 p.

Mitchell, M.D. y M.P. Denne. 1997. Variation in density of Picea sitchensis in relation to within-tree trends in tracheid diameter and wall thickness. Forestry (Oxford) 70: 47-60.

Ohta, S. 1972. Studies on mechanical properties of juvenile wood especially of Sugi-wood and Hinoki-wood. Bulletin Kyushu University Forest 45: 1-80.

Panshin, A.J. y C. De Zeeuw. 1980. Textbook of wood technology. 3rd. Ed. McGraw-Hill. Nueva York. 722 p.

Paraskevopoulou, A.H. 1991. Variation of wood structure and properties of Cupressus sempervirens var. horizontalis in natural populations in Greece. IAWA Bulletin new series 12(2): 195-206.

Pumijumnong, N. y W. Park. 1999. Vessel chronologies from teak in Northern Thailand and their climatic signal. IAWA Journal 20(3): 285-294.

Rzedowski, J. 1978. La vegetación de México. Ed. Limusa. México. 432 p.

SAS Institute. 1988. SAS/STAT user's guide, release 6.03 de. SAS Institute Inc., Cary, NC. 956 p. 
Shortle W.C., K.T. Smith, R. Minocha, V.A. Alexeyev y P. Stott. 1995. Similar patterns of change in stemwood calcium concentration in red spruce and Siberian fir. Journal of Biogeography 22: 467-473.

Siddiqi, T.O. 1991. Impact of seasonal variation on the structure and activity of vascular cambium in Ficus religiosa. IAWA Bulletin new series 12(2): 177185.

Torelli, N., K. Ėufar y D. Roviè.1986. Some wood anatomical, physiological and silvicultural aspects of silver fir dieback in Slovenia (NW Yugoslavia). IAWA Bulletin new series 7(4): 343-351.

Vaganov, E.A. 1996. Cells of tree rings reflect the rise in air temperature during this century. Doklady, Biological Sciences 351: 582-584.

Venogupal, N. y K.V. Krishnamurthy. 1987. Seasonal production of secondary xylem in the twig of certain tropical trees. IAWA Bulletin new series 8(1): 31-40.

Weber, U.M. 1997. Dendroecological reconstruction and interpretation of Larch budmoth (Zeiraphera diniana) outbreaks in two central alpine valleys of Switzerland from 1470-1990. Trees: Structure and Function 11: 277-290.

Wilpert, K-von. 1991. Intraannual variation of radial tracheid diameters as monitor of site specific water stress. Dendrochronologia 9: 95-113.

Wimmer, R., M. Grabner, E. Schäy y G. Halbwachs. 1996. How useful is xylem anatomy as an indicator for $\mathrm{SO} 2$ pollution?. IAWA Journal 17(3): 266267.

Xiong, L., N. Okada, T. Fujiwara, S. Ohta y J.G. Palmer. 1998. Chronology development and climate response analysis of different New Zealand pink pine (Halocarpus biformis) tree-ring parameters. Canadian Journal of Forestry Research 28: 566-573. 